Ann. Génét. Sél. anim., I973, 5 (4), 477-493.

\title{
ÉTUDE DES CONDITIONS DE MUTAGENESE DE LA SOUCHE BHK 21/13 \\ POUR LA SÉLECTION DE RÉSISTANTS \\ A LA 5-BROMODEOXYURIDINE

\author{
OU A LA 8-AZAGUANINE
}

\author{
M. CABOCHE \\ avec la collaboration technique de Ginette GIFFARD \\ Laboratoire de Génétique cellulaire, \\ Centre de Recherches de Toulouse, I. N. R. A., \\ B. P. 12, 31320 Castanet Tolosan
}

\section{RÉSUMÉ}

Les marqueurs génétiques de résistance à la 8-azaguanine et à la 5-bromodéoxyuridine sont employés pour étudier les conditions de mutagénèse de cellules somatiques de Hamster syrien (souche $B H K$ 21/13). Les effets de diverses drogues sur la croissance et l'efficacité d'étalement de la souche sont comparés. Parallèlement le pouvoir mutagène de ces drogues est mesuré. L'éthyle méthane sulfonate et la $\mathrm{N}$-méthyle- $\mathrm{N}^{\prime}$-nitro- $\mathrm{N}$-nitrosoguanidine sont de puissants mutagènes ; la 5-bromodéoxyuridine et le méthyle méthane sulfonate sont actifs à un degré moindre, enfin la caféine est faiblement mutagène.

Le temps d'expression des mutations croît avec la dose de mutagène employée pour induire les mutations. Après la mutagénèse une moyenne de deux divisions cellulaires est nécessaire à l'expression de $50 \mathrm{p}$. roo des mutations induites.

La fréquence de mutation pour la résistance à la 8-azaguanine croît linéairement avec la concentration en mutagène; il n'en est pas de même pour les mutations de résistances à la 5-bromodéoxyuridine. Les implications de ces résultats pour le test du pouvoir mutagène de composés divers et pour la mise au point des conditions standard de mutagenèse sont discutées.

\section{INTRODUC'TION}

Les premières lignées cellulaires porteuses de déficiences enzymatiques héréditaires caractérisées ont été obtenues par prélèvements sur des patients affectés par un syndrome héréditaire (KROOTH et WEINBERG, I96I) ou plus fréquemment par 
sélection directe en culture à partir de lignées cellulaires établies (SzyBaLSKI et Smiтh, 1959). Dans ce dernier cas le caractère héréditaire des déficiences n'a été que rarement mis en évidence. En effet l'adaptation temporaire de souches à certains agents de sélection est un phénomène fréquent, des modifications de perméation non héréditaires pouvant être à l'origine de cette adaptation (MEZGER FREED, I97I).

Deux méthodes permettent d'étudier le mécanisme d'apparition d'une variation phénotypique : d'une part l'apparition d'un phénotype modifié peut être démontrée aléatoire grâce au test de fluctuation (LEA et CoUL,SON, I949) ; cette méthode a été employée en particulier dans l'étude de la résistance à la 8-azaguanine (CHU et MALLING, I969), d'autre part une variation phénotypique aléatoire peut être montrée héréditaire en étudiant l'effet des agents mutagènes sur le taux d'apparition de cette variation (KAO et PucK, I969). La deuxième méthode a été employée dans cette étude ; sa mise au point permet en outre la sélection de variants phénotypiques héréditaires à taux de mutation spontanée très faible donc difficiles à isoler.

Pour mettre au point les conditions de mutagénèse, deux marqueurs génétiques classiques ont été employés : résistance à la 8 -azaguanine ou à la 5-bromodéoxyuridine (LITTLEFIELD et BASILICO, I966). La 8-azaguanine a des effets multiples sur le métabolisme des bases puriques; en particulier elle semble bloquer la synthèse de la guanosine 5'-phosphate (BALIS, I968). La sélection de résistants à cette drogue a été fréquemment effectuée. Les résistants analysés ont généralement une déficience héréditaire en hypoxanthine-guanine-phosphoribosyltransférase. Le gène correspondant est localisé sur le chromosome X chez l'Homme (NABHOLZ et al., I969) et le hamster chinois (WESTERVELD et al., I97I). La 5-bromodéoxyuridine est incorporée dans I'ADN (TAYLOR, I968) cette incorporation induit des erreurs au cours de la replication et des cassures chromosomiques fréquemment léthales. Les souches cellulaires résistantes à la 5-bromodéoxyuridine sont généralement déficientes en thymidine-kinase. Le gène correspondant est autosomique chez l'Homme (Matsuya et $a l .$, I968) et le hamster chinois (WESTERVELD et al., I972).

L'étude des conditions de mutagénèse réalisée avec l'éthyle méthane sulfonate et avec la $\mathrm{N}$-méthyle $\mathrm{N}^{\prime}$-nitro $\mathrm{N}$-nitrosoguanidine utilise ces deux marqueurs génétiques.

\section{MÉTHODES}

\section{A. - Souche}

La souche BHK 21/13 (Stocker et Mac Pherson, 1964) (') provient de l' "American type culture collection " (CCL I0). Elle est cultivée à $36,5^{\circ} \mathrm{C}$ en milieu essentiel minimum (EAGLE, 1959) tamponné $\mathrm{HANKS}_{\mathrm{AN}}$ Tris $\mathrm{HCl} \mathbf{1}^{-2} \mathrm{M} \mathrm{pH} 7,2$ et additionné de sérine, d'asparagine et de pyruvate de sodium (5. I0 ${ }^{-4} \mathrm{M}$ chacun) ainsi que $4 \mathrm{p}$. Ioo de sérum fotal bovin et $6 \mathrm{p}$. Ioo de sérum de veau. Ce milieu permet le clonage de la souche (MEM CL) (CABOCHE, I973).

Une lignée $\left(\mathrm{C}_{3}\right)$ ayant un nombre modal de 43 chromosomes a été isolée après deux clonages successifs dans ce milieu dont le sérum a été préalablement dialysé. Cette lignée a les mêmes caractéristiques de croissance et de sensibilité aux drogues que la souche dont elle provient. Elle est conservée congelée à $-70^{\circ} \mathrm{C}$ dans le milieu de culture complémenté par I $5 \mathrm{p}$. Ioo de glycérol et $20 \mathrm{p}$. 100 de sérum fœtal bovin.

(1) Abréviations : ADN : Acide déoxyribonucléique ; BHK : Baby Hamster kidney ; Tris : Trishydroxyméthyleaminométhane. 


\section{B. - Produits chimiques}

Les milieux de culture, le sérum fœtal bovin et le sérum de veau sont préparés par le laboratoire Eurobio et conservés congelés. La 5-bromodéoxyuridine (A grade), la 2-aminopurine et la 8-azaguanine (B grade) sont des produits Calbiochem. La caféine, le méthyle méthane sulfonate (MMS) et la N-méthyle $\mathrm{N}^{\prime}$-nitro N-nitrosoguanidine (MNNG) sont fournis par Aldrich. L'éthyle méthane sulfonate (EMS) est obtenu chez Sigma et l'aminoptérine chez Schuchardt.

Les mutagènes sont généralement des composés instables et des précautions particulières sont prises pour assurer la conservation des solutions concentrées de ces drogues : la MNNG est dissoute à $4^{\circ} \mathrm{C}$ dans un tampon acétate de sodium 2. IO $^{-3} \mathrm{M}, \mathrm{pH} 5$ (ORKIN et LitTLEFIELD, I97I) conservée congelée à $-20^{\circ} \mathrm{C}$ et directement décongelée par addition de milieu de culture préchauffé au moment de l'emploi. (La drogue perd $40 \mathrm{p}$. Ioo de son pouvoir léthal en restant $\mathrm{I}$ heure à $37^{\circ} \mathrm{C}$ et $\mathrm{pH} 7,2$ et semble donc se dégrader rapidement). L'EMS et le MMS sont dilués dans l'alcool absolu et conservés à $-20^{\circ} \mathrm{C}$. Enfin les solutions concentrées de 5 -bromodéoxyuridine sont protégées de la lumière et congelées. Ces conditions de conservation permettent d'assurer une bonne reproductibilité des résultats des mutagénèses. Les mutagènes employés sont détruits par autoclavage.

\section{C. - Test de sensibilité aux drogues}

Les cellules sont repiquées en MEM CL (500 cellules par bouteille en verre de $25 \mathrm{~cm}^{2}$ de surface d'étalement) et incubées 6 heures à $36,5^{\circ} \mathrm{C}$.

Le milieu contenant la drogue est introduit et les cultures sont incubées 8 jours dans ces conditions. Après fixation à l'acide trichloroacétique $5 \mathrm{p}$. Ioo, les colonies sont comptées après coloration au bleu de Unna.

Lorsque la drogue à tester est un mutagène les cellules sont incubées I 6 heures en sa présence puis lavées avec la solution saline de Hanks et incubées en MEM CL pendant 8 jours.

\section{D. - Conditions de mutagénèse et milieux de sélection}

Un à deux millions de cellules sont étalées en bouteille de verre $\left(75 \mathrm{~cm}^{2}\right.$ de surface d'étalement) en milieu MEM CL. Le mutagène est introduit immédiatement après dilution dans le milieu MEM CL préchauffé, 6 heures après le repiquage et pour une durée de 16 heures. Après mutagénèse les cellules sont lavées et le milieu périodiquement renouvelé au cours de la croissance. 5 jours après la fin de la mutagénèse les cellules sont repiquées dans le milieu de sélection (MEM.S, milieu de clonage où la sérine et l'asparagine sont remplacées par l'ensemble des acides aminés non essentiels apportés à la concentration de $\left.10^{-2} \mathrm{M}\right)$.

Pour la sélection de résistants à la 8 -azaguanine, $1 \mathrm{I}^{5}$ cellules sont repiquées par bouteille de $50 \mathrm{~cm}^{2}$ de surface d'étalement et le milieu de sélection contenant $\mathrm{Io} \mu \mathrm{g} / \mathrm{ml}$ de drogue est introduit I 6 heures après repiquage. Pour la sélection de résistants à la 5 -bromodéoxyuridine 2 . 10 ${ }^{5}$ cellules sont repiquées par bouteille et directement soumises à la sélection en MEM. S contenant $25 \mu \mathrm{g} / \mathrm{ml}$ de drogue. Les milieux de sélection sont renouvelés 2,4 et 7 jours après repiquage et les colonies sont comptées de 8 à I I jours après le début de la sélection.

Les colonies testées sont prélevées par grattage avec l'extrémité d'une pipette pasteur. Les clones mutants sont repiqués et entretenus dans le milieu de sélection en présence de drogue afin d'éliminer les révertants éventuels.

Les fréquences de mutation $T_{M}$ sont calculées en tenant compte de l'efficacité d'étalement du mutant dans les conditions de sélection.

$$
T_{M}=\frac{\text { Nombre de colonies résistantes après sélection }}{\begin{array}{l}
\text { Efficacité d'étalement du mutant } \\
\text { dans les conditions de sélection }
\end{array} \times \begin{array}{l}
\text { Nombre de cellules } \\
\text { soumises à sélection }
\end{array}} .
$$

\section{RÉSULTATS}

\section{A. - Conditions de sélection des résistants à la 8-azaguanine et à la 5-bromodéoxyuridine}

A partir de la souche $\mathrm{C} 3$ dite " sauvage " des clones résistants à la 8-azaguanine ont été isolés après mutagénèse. Ces clones n'ont pas d'activité hypoxanthine guaninephosphoribosyltransférase, sont résistants à la 8 -azaguanine Io $\mu \mathrm{g} / \mathrm{ml}$ même après 


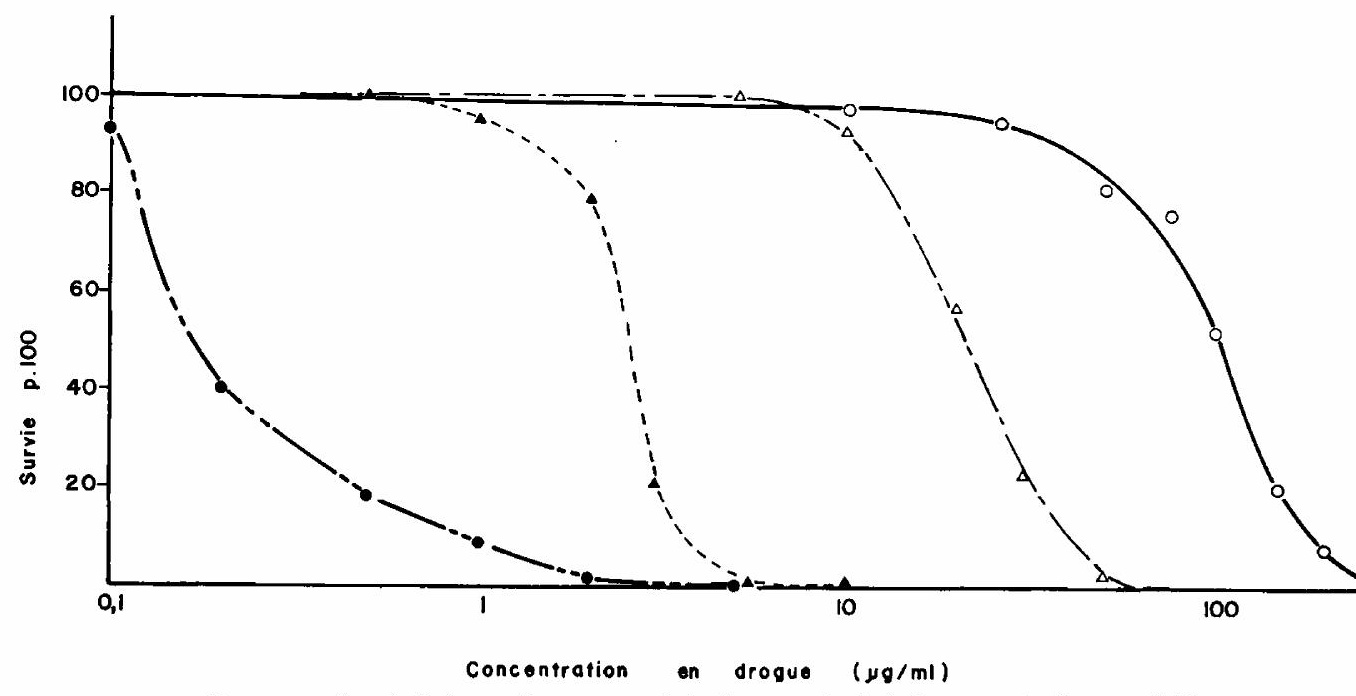

FIG. I. - Survie de la souche sauvage et de clones mutants à des concentrations variables en drogues dans les conditions d'efficacité d'étalement

-- - - - $\mathrm{C}_{3}$, survie à des concentrations variables en 5-bromodéoxyuridine;

$\mathrm{O}-\mathrm{O}_{3}, \mathrm{E} 8, \mathrm{BU}_{3}$, clone résistant à la 5-bromodéoxyuridine. Survie à des concentrations variables en 5-bromodéoxyuridine;

$\mathrm{C}_{3}$, survie à des concentrations variables en 8 -azaguanine ;

( guanine.

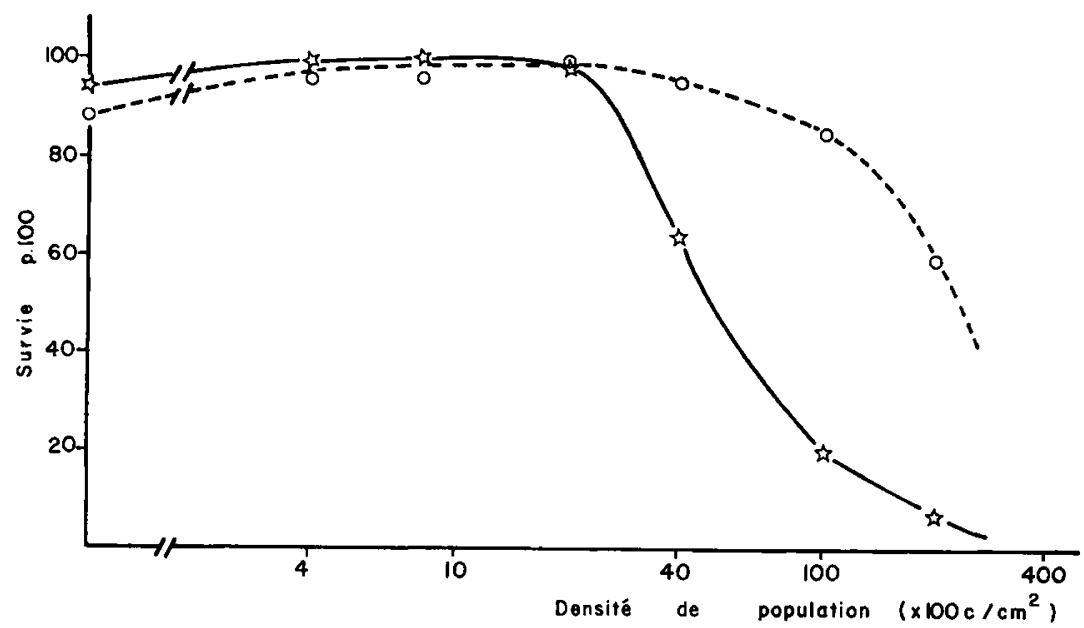

FIG. 2. - Effet de la densité de population en souche sauvage sur la survie du mutant dans les conditions de sélection

La souche mutante est repiquée avec la souche sauvage à concentration cellulaire variable. Les cultures sont soumises à sélection $6 \mathrm{~h}$ après repiquage sans renouvellement du milieu de sélection et les colonies sont fixées et comptées ro jours après le repiquage.

$\star$ —— Io $\mathrm{c} / \mathrm{cm}^{2}$ de la souche $\mathrm{C}_{3}$, ElAGl, résistante à la 8 -azaguanine et concentration variable en souche sauvage $\mathrm{C}_{3}$. Sélection en MEM S contenant ro $\mu \mathrm{g} / \mathrm{ml}$ de 8 -azaguanine ;

$0-. . .-0$ roc/ $\mathrm{cm}^{2}$ de la souche $\mathrm{C}_{3}, \mathrm{E} 8, \mathrm{BU} 3$, résistante à la 5-bromodéoxyuridine et concentration variable en souche sauvage $C_{3}$. Sélection en MEM S contenant $25 \mu \mathrm{g} / \mathrm{ml}$ de 5 -bromodéoxyuridine. 
5 repiquages successifs en absence de drogue et sont incapables de prolifération en milieu HAGT (MEM.CL complémenté avec hypoxanthine $\mathrm{IO}^{-4} \mathrm{M}$, thymidine ${ }^{10^{-5}} \mathrm{M}$ glycine $5 \cdot 10^{-4} \mathrm{M}$ et aminoptérine $10^{-5} \mathrm{M}$ ) (CABOCHE, données non publiées).

La comparaison des seuils de survie des souches résistantes et de la souche $\mathrm{C}_{3}$ permet de définir la concentration optimale en drogue de sélection des résistants : la souche sauvage n'est tuée qu'à concentration en drogue supérieure ou égale à $5 \mu \mathrm{g} / \mathrm{ml}$ (fig. 3).

A la concentration de $20 \mu \mathrm{g} / \mathrm{ml}$ l'efficacité d'étalement du mutant est diminuée et ses colonies plus petites indiquent un effet toxique secondaire de la drogue. Une concentration finale de $\mathrm{Io} \mu \mathrm{g} / \mathrm{ml}$ a été retenue.

A forte densité de population en présence de drogue le mutant est tué par le sauvage (fig. 2). Cet effet toxique dû à la coopération métabolique entre cellules (FUJIMOTO, I97I) est supprimée à une concentration cellulaire inférieure ou égale à 2 ooo cellules $/ \mathrm{cm}^{2}$ (fig. 2 ). Aux faibles densités de population l'efficacité d'étalement du mutant est peu affecté par la présence du sauvage (effet layer, PUCK, I956) et voisine de l'efficacité d'étalement de la souche $\mathrm{C}_{3}$. La fréquence de mutation induite par mutagénèse est calculée en tenant compte de l'efficacité d'étalement de la culture soumise à la sélection.

Des clones résistants à la 5-bromodéoxyuridine $25 \mu \mathrm{g} / \mathrm{ml}$ ont été obtenus après mutagénèse de la souche sauvage. Ces clones n'ont pas d'activité thymidine kinase et sont tués en milieu HAGT, ils sont très stables et conservent leurs caractéristiques de résistance en absence de drogue (САBOchE, données non publiées).

En présence de 5-bromodéoxyuridine les courbes de survie de la souche $\mathrm{C} 3$ et des clones résistants sont très décalées (fig. I). Bien que la survie de la souche $\mathrm{C} 3$ décroisse exponentiellement à partir d'une concentration en 5-bromodéoxyuridine de $0,02 \mu \mathrm{g} / \mathrm{ml}$, des concentrations en drogues supérieures à $10 \mu \mathrm{g} / \mathrm{ml}$ sont nécessaires à l'élimination complète du sauvage. Une concentration finale de $25 \mu \mathrm{g} / \mathrm{ml}$ permet de tuer la souche $\mathrm{C} 3$ et les résistants partiels dans les conditions de sélection.

L'efficacité d'étalement du résistant dans le milieu de sélection contenant $25 \mu \mathrm{g}$ / $\mathrm{ml}$ de drogue est peu affectée par la présence du sauvage jusqu'à des concentrations supérieures à Io 000 cellules $/ \mathrm{cm}^{2}$. Une concentration de 5000 cellules $/ \mathrm{cm}^{2}$ a été retenue pour la sélection des résistants (fig. 2) car aux densités de populations plus élevées l'épuisement du milieu dans les conditions de sélection devient trop rapide.

\section{B. - Détermination de l'effet létal des mutagènes}

Deux parties peuvent être distinguées dans chaque courbe de survie (fig. 3) : un palier de résistance à l'action létale du mutagène, plus ou moins marqué et une zone de décroissance exponentielle de la survie en fonction de la concentration en mutagène. La partie exponentielle commune à toutes les courbes de survie, permet de comparer quantitativement l'effet létal des mutagènes (KAO et PUCK, I969). L'accroissement de concentration en mutagène qui réduit la survie dans le rapport $1 / \mathrm{e}$ dans cette partie exponentielle de la courbe de survie, est appelé dose létale $\left(D_{0}\right)$.

Les courbes diffèrent par leur palier de résistance à l'effet létal du mutagène : ce palier absent pour la MNNG est considérable pour le MMS ; un seuil de concentration en mutagène doit être dépassé dans ce dernier cas pour que son effet létal se manifeste (fig. 3). 


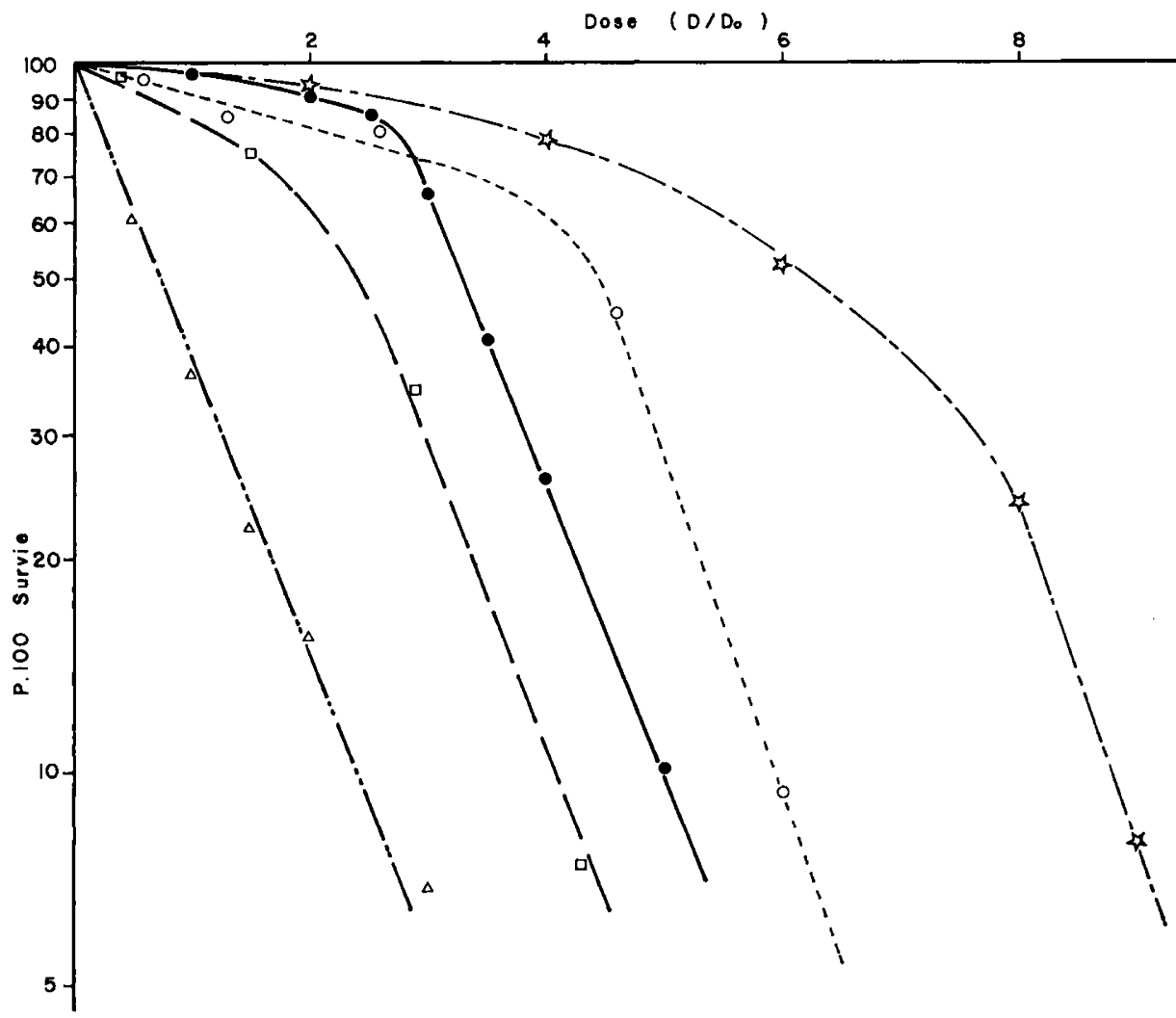

FIG. 3. - Courbe de survie à des concentrations croissantes en mutagène

Les cellules étalées dans les conditions de clonage (ro $\mathrm{c} / \mathrm{cm}^{2}$ ) sont soumises à l'action des mutagènes pendant 16 heures puis incubées en MEM CL. Les résultats sont exprimés en taux de survie par rapport au témoin non traité. $\mathrm{D}_{0}=$ accroissement de concentration en mutagène qui réduit la survie dans le rapport $\mathrm{I} / e$ dans la partie exponentielle de la courbe de survie. $\mathrm{D}=$ concentration ou dose de mutagène dont l'effet toxique est mesuré, exprimée en multiples de $D_{0}$.

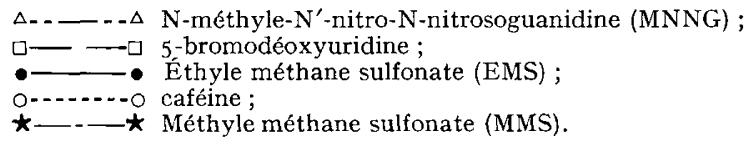


La comparaison des paliers de résistance et les valeurs de $D_{0}$ (tabl. I) montre que chaque agent mutagène agit de façon similaire sur différentes souches cellulaires : aucun palier de résistance n'est détecté pour la MMNG dans de nombreuses souches ; la dose létale est identique pour $B H K 21 / 13$ et pour CHO (KAO et PUCK, I969) dans les mêmes conditions d'incubation en présence d'EMS (tabl. I). Par contre les caractéristiques de la courbe de survie varient énormément d'une drogue à l'autre, certaines étant extrêmement toxiques (MNNG) alors que d'autres n'agissent qu'à concentration très élevée (caféine).

TABLEAU I

Effet létal des mutagènes

\begin{tabular}{c|c|c|c}
\hline Mutagène & $\begin{array}{c}\text { Dose létale }\left(\mathrm{D}_{0}\right) \\
(\mu \mathrm{g} / \mathrm{ml})\end{array}$ & $\begin{array}{c}20 \% \text { de survie } \\
(\mu \mathrm{g} / \mathrm{ml})\end{array}$ & $\begin{array}{c}\text { Position de } \\
\text { l'épaulement }\end{array}$ \\
\hline MNNG & 0,20 & 0,33 & 0 \\
\hline EMS & 100 & 430 & 2,5 \\
\hline MMS & 2,5 & 20 & 6,7 \\
\hline 5-bromodéoxyuridine & 3,5 & 12 & 1,7 \\
\hline Caféine & 1000 & 8000 & 3,8 \\
\hline
\end{tabular}

Les cellules ont été soumises à l'action des mutagènes durant 16 heures dans les conditions d'efficacité d'étalement, 6 heures après repiquage en MEM CL. La position de l'épaulement de la courbe de survie est définie comme l'intersection de l'extrapolation de la partie exponentielle de la courbe de survie avec l'axe des doses de mutagènes (fig. 3). Cette position de l'épaulement est exprimée en multiples de $\mathrm{D}_{0}$.

\section{C. - Temps d'expression des mutations}

Un délai d'expression des mutations provoquées par le mutagène (fig. 4) est généralement observé (CHU et MaLLING, I968). La fréquence de mutation est très faible si la sélection est opérée immédiatement après mutagénèse, elle augmente rapidement avec le temps écoulé entre mutagénèse et sélection puis atteint un palier et décroît progressivement ensuite probablement par élimination des mutants à rythme de prolifération plus lent que la souche sauvage. (MORRow, I972).

Pour une mutagénèse donnée, le temps d'expression moyen des mutations (Te) est défini comme le temps nécessaire à l'expression de $50 \mathrm{p}$. Ioo des mutations obtenues dans les meilleures conditions d'expression, en prenant pour temps zéro l'instant situé au milieu de la mutagénèse. Te augmente avec la concentration en EMS employée au cours de la mutagénèse. Parallèlement le rythme de prolifération cellulaire diminue et le temps de doublement des cultures augmente exponentiellement avec la concentration en mutagène après un palier aux faibles concentrations en EMS (fig. 5). 


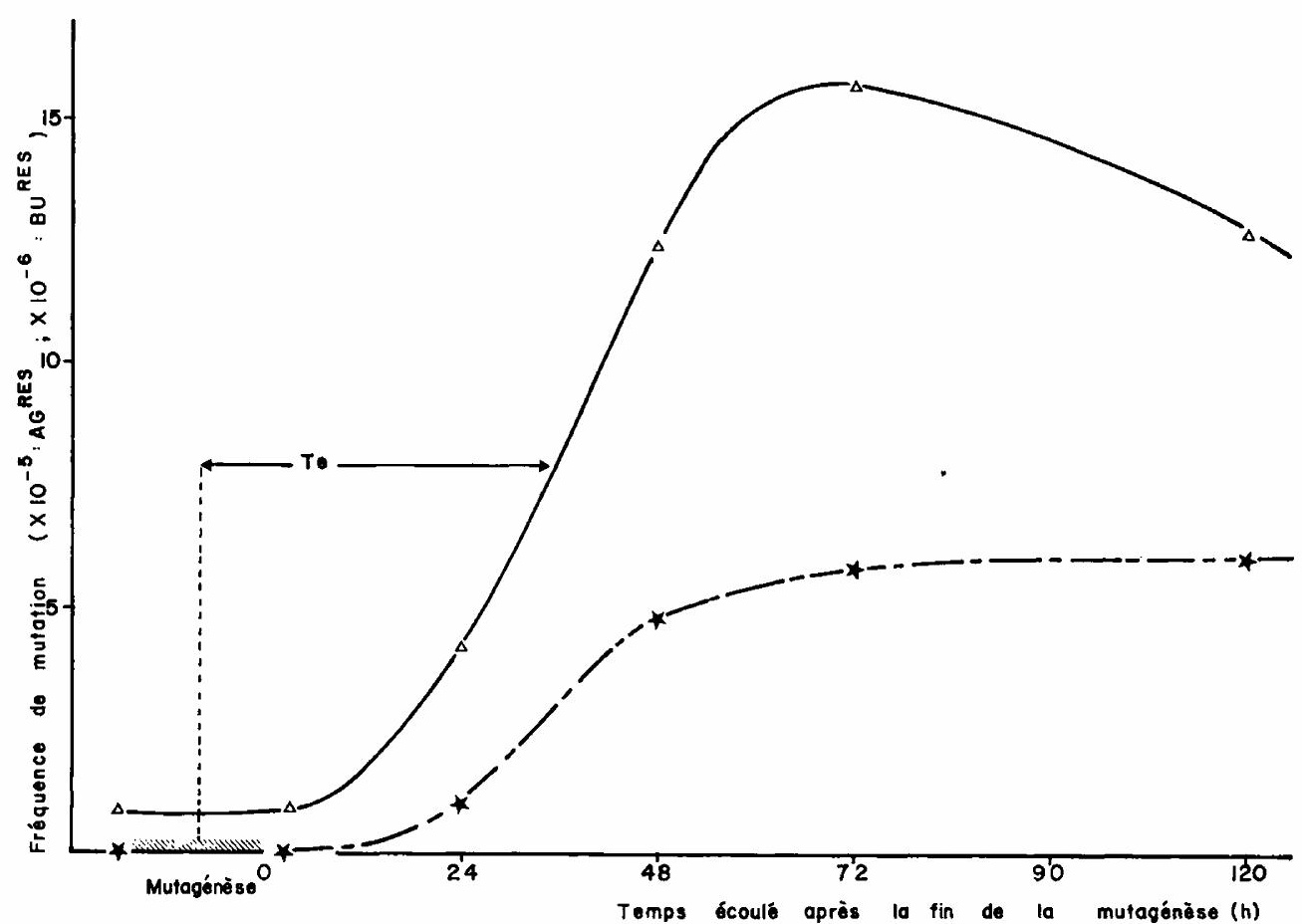

FIG. 4. - Temps d'expression des mutations

Les cellules sont soumises à mutagénèse à l'EMS $250 \mu \mathrm{g} / \mathrm{ml}$ (voir méthodes) puis repiquées après un temps variable de croissance exponentielle en absence d'agent de sélection. 4 à 6 heures après repiquage l'agent de sélection est introduit $\left(5.10^{5}\right.$ cellules soumises à la sélection de résistants à la 8 -azaguanine, ou $2.10^{6}$ cellules soumises à la sélection de résistants à la 5 -bromodéoxyuridine). Il n'est pas tenu compte de l'intervalle de temps écoulé entre repiquage et sélection dans la mesure de Te, temps d'expression de $50 \mathrm{p}$. roo des mutations exprimables. Les deux derniers points expérimentaux correspondent à des cultures repiquées au temps 72 heures en MEM CL une fois supplémentaire avant la sélection.

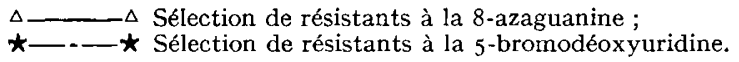




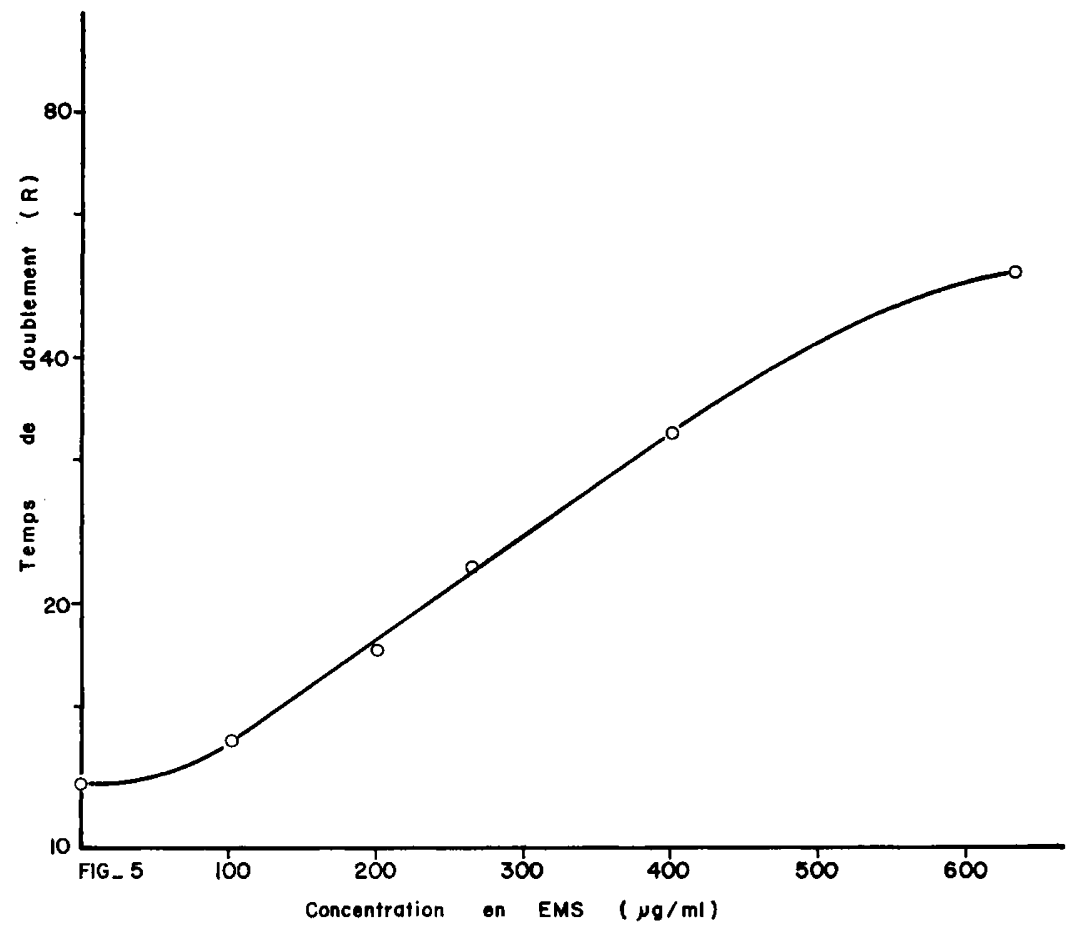

FIG. 5. - Effet de la concentration en mutagène sur la prolifération cellulaire après mutagénèse

Pour chaque concentration en mutagène testée ro bouteilles de $25 \mathrm{~cm}^{2}$ de surface d'étalement contenant $10^{5}$ cellules chacune sont soumises à une mutagénèse de 16 heures, 6 heures après le repiquage.

Toutes les 24 heures les cellules de deux bouteilles sont dissociées et comptées au Coulter Counter. Une croissance exponentielle est observée même aux concentrations élevées en mutagène; le temps de doublement des cultures est déterminé graphiquement sur papier semi-logarithmique.

\section{TABLEAU 7}

Nombre de divisions nécessaires à l'expression des mutations après mutagénèse.

\begin{tabular}{c|c|c|c}
\hline \hline $\begin{array}{c}\text { Mutagénèse } \\
(\mu \mathrm{g} / \mathrm{m})\end{array}$ & $\begin{array}{c}\text { Temps d'expres- } \\
\text { sion moyen Te } \\
\text { des mutations de } \\
\text { résistance à la } \\
5 \text {-bromodéoxy- } \\
\text { uridine (h) }\end{array}$ & $\begin{array}{c}\text { Temps de double- } \\
\text { ment en phase } \\
\text { exponentielle } \\
\text { après mutagénèse } \\
(\mathrm{h})\end{array}$ & $\begin{array}{c}\text { Ne Nombre de } \\
\text { divisions néces- } \\
\text { saires à l'expres- } \\
\text { sion des muta- } \\
\text { tions }\end{array}$ \\
\hline EMS 250 & -44 & 21 & 2,1 \\
\hline EMS 400 & 58 & 31 & 2,2 \\
\hline MNNG 0,5 & -63 & 2,0 \\
\hline
\end{tabular}

Te temps d'expression moyen des mutations représente le temps nécessaire à l'expression de 50 p. 100 des mutations exprimables pour une mutagénèse donnée. $\mathrm{N} e$ est le rapport de $\mathrm{T} e$ au temps de doublement pour une mutagénèse donnée. 
Le rapport de Te au temps de doublement après mutagénèse permet d'estimer le nombre de divisions nécessaires à l'expression des mutations (tabl. 2). Pour les résistants à la 5 -bromodéoxyuridine ce nombre de divisions est identique pour les deux mutagènes testés et indépendant de la concentration en mutagène employée.

\section{D. - Effet de la concentration en mutagène sur le taux de mutation}

L'EMS, plus stable que la MNNG et permettant donc des manipulations plus reproductibles a été employé dans cette étude. L'effet de la concentration en mutagène sur la fréquence de mutation ne peut être étudié que dans des conditions où toutes les mutations induites sont exprimées. Les cellules ont donc été soumises à sélection 6 jours après mutagénèse (avec un repiquage intermédiaire) afin de permettre 1'expression des mutations induites aux fortes concentrations en EMS. La fréquence de mutation pour la résistance à la 8-azaguanine croît linéairement avec la concentration en EMS (fig. 6). Cette courbe comparée à celle de l'effet létal du mutagène montre que 1'EMS est mutagène aux concentrations où il n'est pas toxique. Une croissance linéaire de la fréquence de mutation en fonction de la concentration en mutagène est aussi observée pour la sélection de résistants à la 8 -azaguanine $20 \mu \mathrm{g} /$ $\mathrm{ml}$.

La fréquence de mutation pour la résistance à la 5-bromodéoxyuridine ne crôit pas linéairement avec la concentration en EMS. La courbe peut être lissée correctement par une cubique : 1'obtention d'un grand nombre de résistants à la 5-bromodéoxyuridine nécessite donc l'emploi de fortes concentrations en mutagène.

\section{E. - Effet de la concentration en agent de sélection sur la fréquence de mutation}

La sélection de résistants à la 8-azaguanine pose des problèmes particuliers dus à la faible différence entre les seuils de résistance du sauvage et des mutants (fig. I). Le temps d'expression après mutagénèse influe sur la forme des courbes de survie (fig. 7) ; lorsque ce temps est court, une quantité importante de clones à croissance ralentie survit à la sélection en 8 -azaguanine $\mathrm{Io} \mu \mathrm{g} / \mathrm{ml}$; ces clones ne sont pas résistants à la drogue après repiquage. Tout se passe comme si le mutagène ralentissant la croissance de certains clones, leur permet de métaboliser moins activement la 8-azaguanine, modifiant ainsi la pente de la courbe de survie du sauvage après mutagénèse. Si par contre le temps d'expression est suffisamment long, les pseudo-résistants disparaissent des cultures et la sélection en MEM.S, 8-azaguanine ro $\mu \mathrm{g} / \mathrm{ml}$, ne laisse subsister que des clones conservant leurs caractéristiques de résistance après repiquage : dans ces conditions d'expression la fréquence de mutation varie peu avec la concentration en agent de sélection. Dans tous les cas des clones résistants sélectionnés en 8 -azaguanine $20 \mu \mathrm{g} / \mathrm{ml}$ conservent leurs caractéristiques de résistance après repiquage : les pseudo-résistants sont donc éliminés à cette concentration de sélection ; en contrepartie la survie du mutant est elle-même diminuée dans ces conditions. La sélection de résistants à la 5 -bromodéoxyuridine ne pose pas ce type de problème : les fréquences de mutation après sélection en MEM.S, 5-bromodéoxyuridine 25 ou $50 \mu$ sont comparables quelque soit le temps d'expression. 


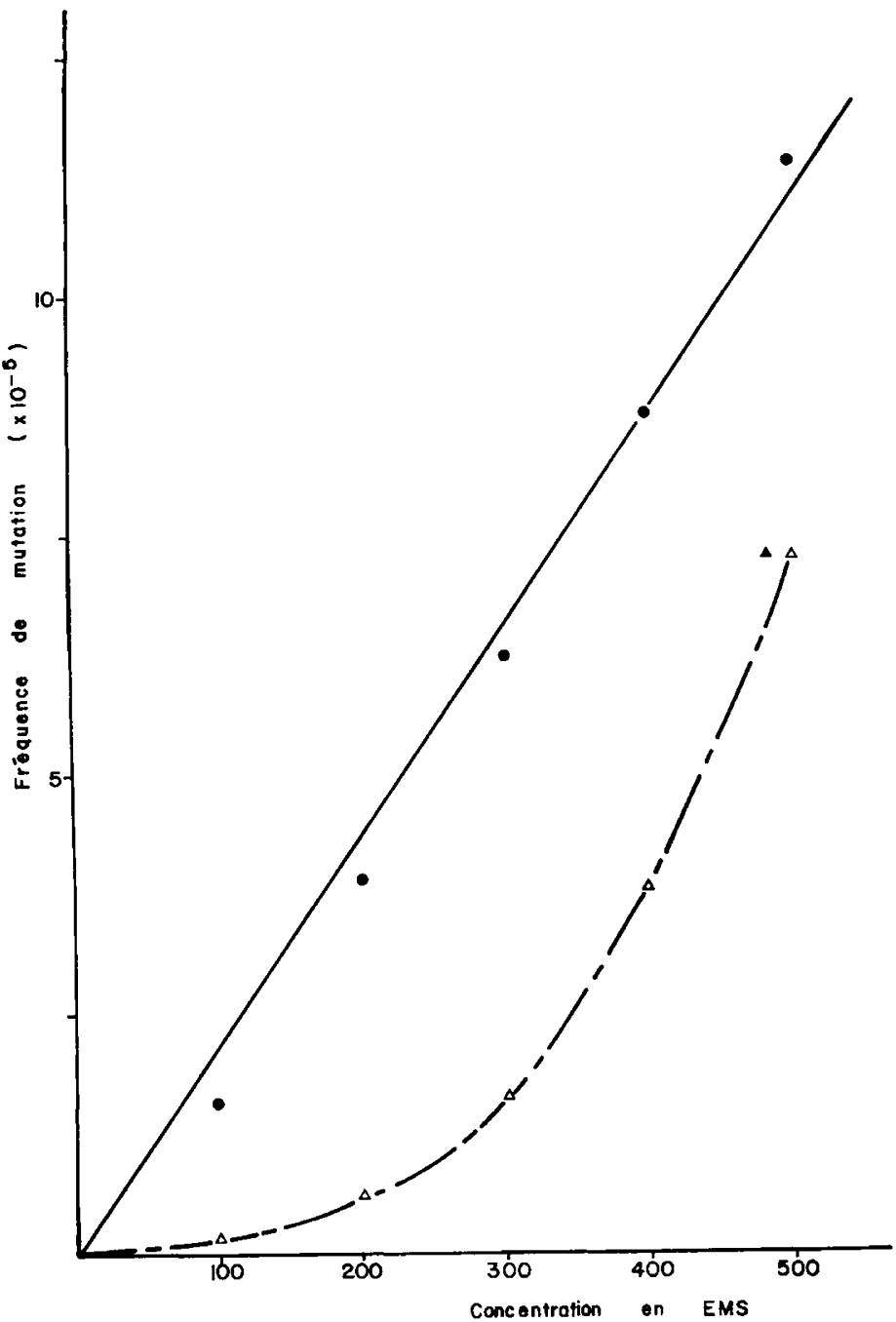

FIG. 6. - Effet de la concentration en mutagène sur le taux de mutation

Les clones résistants à la 8 -azaguanine ro $\mu \mathrm{g} / \mathrm{ml}$ et à la 5 -bromodéoxyuridine $25 \mu \mathrm{g} / \mathrm{ml}$ ont été sélectionnés après mutagénèse à l'EMS dans les conditions décrites dans les méthodes. Un temps d'expression de 6 jours avec un repiquage intermédiaire en MEM CL permet l'expression de toutes les mutations et l'élimination des pseudo-résistants à la 8-azaguanine (paragraphe.E). Les fréquences de mutations $\mathrm{T}_{\mathbf{M}}$ sont déterminées pour chaque concentration en mutagène après sélection effectuée sur $10^{6}$ cellules. (résistants à la 8 -azaguanine) ou $2.10^{6}$ cellules (résistants à la 5-bromodéoxyuridine) (voir tabl. 3 ). Les mêmes types de courbes ont été obtenus dans 3 manipulations indépendantes.

$\Delta \_-\Delta T_{M}$ pour la sélection de résistants à la 8 -azaguanine ro $\mu \mathrm{g} / \mathrm{ml}$;

- — $-T_{M}$ pour la sélection de résistants à la 5-bromodéoxyuridine $25 \mu \mathrm{g} / \mathrm{ml}$. 


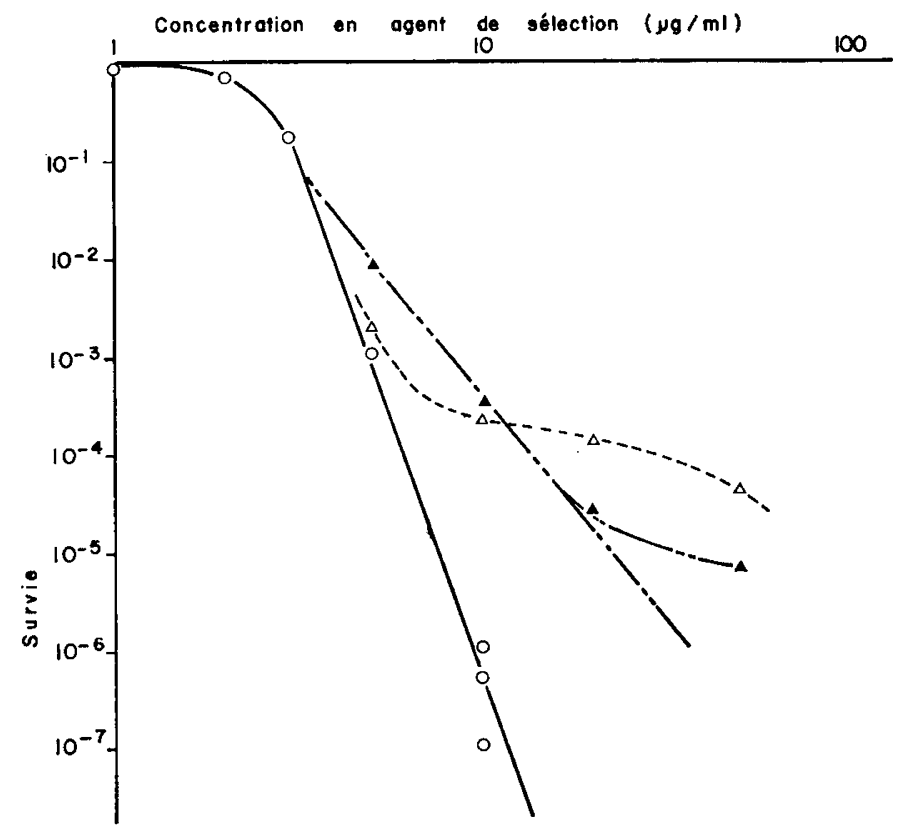

FIG. 7. - Effet de la concentration en agent de sélection sur la survie de la souche sauvage soumise ou non à mutagénèse

Les résistants à des concentrations variables en 8-azaguanine sont sélectionnés dans les conditions décrites aux méthodes. Les résultats sont exprimés en taux de survie par rapport aux cultures non soumises à sélection. On remarque la modification de la pente de la courbe de survie de la souche sauvage après mutagénèse si le temps d'expression des mutations est trop court.$$
\text { o- } 0 \text { Témoin sans mutagénèse ; }
$$$$
\Delta-\ldots \text { Mutagénèse EMS } 300 \mu \mathrm{g} / \mathrm{ml}, \mathrm{r} 6 \mathrm{~h} \text {, temps d'expression } 24 \mathrm{~h} \text {; }
$$$$
\Delta \ldots . . . \Delta \text { Mutagénèse EMS } 300 \mu \mathrm{g} / \mathrm{ml} \text {, I6 h, temps d'expression } \mathrm{I} 20 \mathrm{~h} \text {. }
$$ 
F. - Taux de mutation pour divers mutagènes (tab1. 3).

Un certain nombre de mutagènes ont été testés simultanément pour leur capacité d'induction de mutants résistants à la 8-azaguanine et à la 5-bromodéoxyuridine. Il n'a pas été obtenu jusqu'ici de résistants spontanés à la 5-bromodéoxyuridine et ceci avec des sélections portant sur $4 \cdot 10^{6}$ cellules effectuées à plusieurs reprises. L'apparition de ce marqueur après mutagénèse est donc un critère excellent de pou-

TABLEAU 3

Fréquence de mutation induite après mutagenèse

\begin{tabular}{|c|c|c|c|}
\hline Agents de sélection & $\begin{array}{l}\text { 5-bromodéoxyuridine } \\
25 \mu \mathrm{g} / \mathrm{ml}\end{array}$ & \multicolumn{2}{|c|}{$\begin{array}{l}\text { 8-azaguanine } \\
10 \mu \mathrm{g} / \mathrm{ml}\end{array}$} \\
\hline Mutagène & $\left({ }^{\mathbf{1}}\right)$ & $(\mathbf{1})$ & $\left({ }^{2}\right)$ \\
\hline Témoin sans mutagène & $<5 \cdot 10^{-7}\left({ }^{3}\right)$ & $4,3 \cdot 10^{-6}\left({ }^{3}\right)$ & - \\
\hline EMS $400 \mu \mathrm{g} / \mathrm{ml}$ & $1,8 \cdot 10^{-5}$ & $2,4 \cdot 10^{-4}$ & $6,0 \cdot 10^{-5}$ \\
\hline MNNG $0,5 \mu \mathrm{g} / \mathrm{ml}$ & $3,6 \cdot 10^{-5}$ & $1,4 \cdot 10^{-4}$ & $5,7 \cdot 10^{-5}$ \\
\hline $\begin{array}{l}\text { 5-bromodéoxyuridine } \\
10 \mu \mathrm{g} / \mathrm{ml}\end{array}$ & $3,6 \cdot 10^{-6}$ & $6,8 \cdot 10^{-5}$ & $2,4 \cdot 10^{-5}$ \\
\hline Caféine $3000 \mu \mathrm{g} / \mathrm{ml}$ & $<5 \cdot 10^{-7}$ & $6 \cdot 10^{-6}$ & $6 \cdot 10^{-7}$ \\
\hline $\begin{array}{l}\text { 2-aminopurine } \\
50 \mu \mathrm{g} / \mathrm{ml}\end{array}$ & $<5 \cdot 10^{-7}$ & $2,3 \cdot 10^{-6}$ & - \\
\hline
\end{tabular}

Les cellules sont soumises à mutagenèse et sélectionnées dans les conditions décrites aux méthodes, $10^{6}$ cellules ont été soumises à sélection une ou plusieurs fois dans chaque test.

(1) Fréquence de mutation: $T_{M}$

$\mathrm{T}_{\mathbf{M}}=\frac{\text { Nombre de colonies obtenues après sélection }}{\begin{array}{c}\text { Nombre de cellules soumises } \\ \text { à sélection }\end{array} \times \begin{array}{c}\text { Efficacité d'étalement des cellules } \\ \text { soumises à sélection }\end{array}}$.

$\left({ }^{2}\right)$ Fréquence de mutation par dose létale :

$\frac{T_{M} \times D_{0}}{\text { Concentration en mutagène employée }}$.

${ }^{(3)}$ Dans les expériences témoins les résultats ne représentent pas un taux de mutation spontanée par cellule et par génération, mais une mesure du nombre des mutants spontanés présents dans les cultures non soumises à mutagenèse.

voir mutagène. Le taux de mutation spontanée pour la résistance à la 8-azaguanine n'a pas été systématiquement déterminé par test de fluctuation. La fréquence de mutation est simplement contrôlée à chaque mutagenèse en conservant une culture non traitée.

Annales de Génétique animale. - 1973. 


\section{DISCUSSION}

\section{A. - Détermination de la fréquence des mutations induites}

Plusieurs conditions sont nécessaires à la détermination quantitative des fréquences de mutations induites par mutagénèse :

- Le système de sélection ne doit laisser proliférer que des clones mutants.

- Le taux de mutation spontanée doit être négligeable par rapport à la fréquence des mutations induites.

- Toutes les mutations induites par mutagénèse doivent être exprimées au début de la sélection.

- Enfin la survie du mutant ne doit pas être modifiée par la croissance du sauvage dans les conditions de sélection.

Les résultats expérimentaux montrent que pour respecter ces conditions un certain nombre de précautions doivent être prises :

En premier lieu la souche doit être clonée ou passée en milieu HAGT afin d'éliminer la présence de résistants éventuels ; pour chaque mutagénèse, une sélection témoin sur des cellules non traitées doit être effectuée afin de déterminer la présence éventuelle de résistants spontanés. L'expression correcte des mutations induites est le problème majeur de la détermination des fréquences de mutation : plus la concentration en mutagène employé est élevée, plus ce temps d'expression doit être long. Si l'on emploie de grandes quantités de cellules pour effectuer les mutagénèses, afin d'obtenir un nombre important de mutants indépendants après sélection, les cultures soumises à l'action $\mathrm{du}$ mutagène arrivent rapidement à confluence. Un repiquage intermédiaire est alors nécessaire entre mutagénèse et sélection si l'on veut assurer un temps d'expression suffisant des mutations induites (ORKIN et LITTLEFIELD, i97 ; Chu et MALLING, I969).

\section{B. - Temps d'expression des mutations induites}

Le mécanisme d'action des mutagènes a été le plus souvent étudié chez les bactéries et les virus. Bien que le mécanisme d'action ne soit pas nécessairement identique pour les cellules de mammifères (ORKIN et LITTLEFIELD, I97I) il est probable que les agents alkylants que sont 1'EMS et la MNNG provoquent des mutations en induisant des erreurs d'appariement au niveau de l'ADN au cours de la replication (FREESE, I963). Deux replications sont donc théoriquement nécessaires à l'apparition des mutations.

La thymidine kinase est un enzyme synthétisé périodiquement au cours du cycle cellulaire, l'activité enzymatique étant très faible au moment de la mitose (BRENT, I965), l'apparition d'une mutation au niveau du gène de structure de la thymidine kinase est donc exprimée au plus tard dès la mitose qui suit cette mutation. L'ensemble des événements conduisant théoriquement à 1'expression d'une mutation de résistance à la 5-bromodéoxyuridine nécessite donc deux phases de synthèse d'ADN suivies d'une mitose, soit une moyenne de deux cycles cellulaires si l'on suppose que l'alkylation par le mutagène s'effectue sur des cultures en phase exponentielle.

Cette hypothèse est compatible avec les résultats obtenus chez $\mathrm{BHK} 2 \mathrm{I} / \mathrm{I} 3$. 
Le nombre de cycles cellulaires effectués entre la mutagenèse et l'apparition de $50 \mathrm{p}$. Ioo des mutations induites est indépendant de la concentration en mutagène et voisin de deux cycles. Le paramètre important dans l'expression des mutations n'est donc pas le temps écoulé mais plutôt le nombre de cycles cellulaires effectués entre mutagenèse et sélection.

Les conditions d'expression des mutations de résistance à la 8-azaguanine sont comparables à celles observées pour la résistance à la 5-bromodéoxyuridine. Leur étude est compliquée par l'apparition de pseudo-résistants après mutagenèse et rend les résultats plus difficiles à interpréter.

\section{C. - Comparaison des effets des mutagènes}

Trois hypothèses peuvent expliquer l'existence d'un palier dans la courbe de survie à l'action de doses croissantes de mutagènes (KAO et PUCK, I959).

- Une concentration minimale en drogue est nécessaire pour permettre la pénétration de cette drogue jusqu'à son site d'action.

- Plusieurs structures cellulaires doivent être inactivées pour provoquer la mort de la cellule.

- Les processus de réparation masquent l'effet létal du mutagène ils doivent être saturés pour que se manifeste cet effet létal. La première hypothèse est contredite par les résultats expérimentaux : même aux concentrations où il n'est pas toxique 1'EMS est mutagène et donc pénètre bien jusqu'au niveau de 1'ADN. L'effet létal des mutagènes est en étroite corrélation avec l'apparition de cassures ou d'anomalies chromosomiques (KAO et PUCK, I969) ; ces cassures peuvent être réparées, et il est probable que leur multiplication doit simultanément s'accompagner d'erreurs de réparation. Les deux dernières hypothèses associées (cassures multiples et réparation) peuvent donc apporter une interprétation à la forme des courbes de survie.

Alors que le taux de mutation pour la résistance à la 8-azaguanine croît linéairement avec la concentration en mutagène, il n'en est pas de même pour le taux de mutation pour la résistance à la 5 -bromodéoxyuridine. Cette différence peut être interprétée de la façon suivante ; un seul événement mutationnel est a priori nécessaire à la suppression de l'activité hypoxanthine-guanine phosphoribosyltransférase, cet enzyme étant codé par un gène porté par le chromosome $\mathrm{X}$ présent à un seul exemplaire chez $\mathrm{BHK} 2 \mathrm{I} / \mathrm{I} 3$; par contre plusieurs événements mutationnels sont nécessaires à l'inactivation héréditaire de la thymidine kinase dont le gène de structure, autosomal, est certainement présent à plusieurs exemplaires.

L'emploi du marqueur de résistance à la 8-azaguanine permet de comparer de façon quantitative les pouvoirs mutagènes de différentes drogues car le taux de mutation étant proportionnel à la dose létale pour de nombreux mutagènes (HUBERMAN et HEIDELBERGER, I972), on peut calculer pour chaque drogue son pouvoir mutagène par dose létale. L'EMS et la MNNG sont les plus puissants mutagènes testés ; il en est de même pour de nombreuses souches cellulaires. La 5-bromodéoxyuridine est mutagène à un moindre degré. Ceci peut expliquer la relative facilité d'obtention du marqueur de résistance à la 5-bromodéoxyuridine par sélection à concentration croissante en drogue, la drogue induisant sa propre mutation de résistance. La caféine provoque des cassures, son pouvoir faiblement mutagène peut donc avoir pour origine l'augmentation du taux de mutations spontanées. 
La technique de mesure du pouvoir mutagène est sensible et permet de déceler des fréquences de mutations induites faibles $\left(>I^{-6}\right)$. Le dosage du pouvoir mutagène de certains adjuvants intervenant directement comme polluants dans l'alimentation (colorants, stabilisants, arômes, pesticides, desherbants) peut donc être étudié quantitativement par cette méthode.

Reçu pour publication en août 1973.

\section{REMERCIEMENTS}

Nous remercions le Dr Louarn du Laboratoive de Génétique de la Faculté des Sciences de Toulouse dont les critiques nous ont été précieuses pour la rédaction du manuscrit.

\section{SUMMARY}

STUDY OF MUTAGENESIS CONDITIONS OF BHK 21/13

CELL STRAIN FOR THE SEIECTION OF 5-BROMODEOXYURIDINE OR 8-AZAGUANINE RESISTANCE GENETIC MARKERS

8-azaguanine and 5-bromodeoxyuridine resistance genetic markers are used to study mutagenesis conditions of BHK 2I $\mathrm{S}_{3} 3$ cell strain. Different drug effects upon growth and plating efficiency are compared and their mutagenic action is measured.

Ethyl methane sulfonate and $\mathrm{N}$-methyl $\mathrm{N}^{\prime}$-nitro- $\mathrm{N}$-nitrosoguanidine are potent mutagens; 5-bromodeoxyuridine and methyl methane sulfonate are less active, cafeine is a poor mutagen.

Expression time of mutation is higher at bigger mutagen hit. An average of two cell divisions are necessary after mutagenesis for the expression of $50 \mathrm{p}$. Ioo of induced mutations.

Mutation frequency for induced 8-azaguanine resistance grows linearly with mutagen concentration. This is not true for induced 5-bromodeoxyuridine resistance mutations. discussed.

Those results availability for routine measurements of different drugs mutagenic action are

\section{RÉFÉRENCES BIBLIOGRAPHIQUES}

BALIS M. E., 1968. Antagonists and nucleic acids. North Holland publishing company Amsterdam 39-4r.

Brent T. P., Butler J. A. V., Crathorn A. R., I965. Variations in phosphokinase activities during the cell cycle in synchronous populations of Mela cells. Nature, 207, I76-I77.

CABOche M., I973. Conditions de clonage et de culture en suspension de la souche $\mathrm{BHK} 2 \mathrm{r} / \mathrm{I} 3 \mathrm{de}$ hamster syrien. Sensibilité des cellules aux inhibiteurs du métabolisme des acides nucléiques. Ann. Biol. Anim. Bioch. Biophys. (à paraître).

$\mathrm{CHu}$ E. M. Y., Malling H. V., I968. Mammalian cell genetics II. Chemical induction of specific locus mutations in chinese hamster cells in vitro. Proc. Nat. Acad. Sci. 61, 1306-1312.

FREese E. I963. Molecular genetics edited by Taylor J. M. Academic Press New York London part I-207.

Fujimoto W. Y., Subak-Sharpe J. H., Seegmiller J. E., I97I. Hypoxanthine-guanine phosphoribosyl transférase deficiency : chemicals agents for mutant cultured fibroblasts in mixed and heterozygote cultures. Proc. Nat. Acad. Sci. 68, I5 I6-I5 19.

Huberman E., Heidelberger C., r972. The mutagenicity to mammalian cells of pyrimidine nucleoside analogues. Mutation res. 14, 130-132. 
KAo F. T., PuCK T. T., I969. Genetics of somatic mammalian cells IX quantitation of mutagenesis by physical and chemical agents. J. cellular physiol. 74, 245-258.

KRooth R. S., Weinberg A. N., I96I. Studies of cell lines developped from tissues of patients with galactosemia. J. expl. Med. 115, 317-327.

LeA, Coulson C. A., I949. The distribution of the number of mutants in bacterial populations. J. Genetics $49,264-285$.

LitTlefield J. W., I964. Selection of hybrids from mating of fibroblasts in vitro and their presumed recombinants. Science, 145, 709.

Littlefield, Y. M., Basilico C., r966. Infection of thymidine kinase deficient BHK polyoma cells. Nature, 211, 250.

Matsuya Y., Green M., Basilico C., Ig68. Properties and uses of human-mouse hybrid cell lines. Nature 220, II99-1202.

MEZGer-FreED L., I97I. Puromicin resistance in haploid and heteroploid frog cells : gene or membrane determined. J. Cell. Biol. 51, 742-75r.

Morrow Y., r972. Population dynamics of purine and pyrimidine analog sensitive and resistant mammalian cells grown in culture. Genetics 71, 429-438.

Nabholz M., Mrggiano V., Bodmer W., I969. Genetic analysis with human-mouse somatic cell hybrids. Nature 223, 358-363.

ORkin S. H., LITTLEFIELD Y W., I97I $a$. Nitrosoguanidine mutagenesis in synchronized hamster cells. Expl. Cell. Res. 66, 69-74.

Puck T. T., Marcus P. I., Cieciura S. J., 1956. Clonal growth of mammalian cells in vitro : growth characteristics of colonies from single HELA cells with and without a "feeder "layer. J. Expl. Med. 103, 273-283.

Stocker M., Mac Pherson I., I964. Syrian hamster fibroblast cell line BHK 2 I and its derivates. Nature, 203, I355-I357.

SzYBalski W., Smith J., I959. Genetics of human cell lines I. 8-azaguanine resistance, a selective " single step" marker. Proc. Soc. Expl. Biol. Med., 101, 662-666.

TAYLOR J. H., I968. Rates of chain growth and units of replication in DNA of mammalian chromosomes. J. mol. Biol., 31, 579-594.

Westerveld A., Visser R. P., Freeke M. A., r97r. Evidence for linkage between glucose 6-phosphate dehydrogenase and hypoxanthine-guanine-phosphorybosyltransferase loci in chinese hamster cells. Biochem. Genetics 5, 59I-599.

Westerveld A., Visser R. P., Freecke M. A., Bootsma D., I972. Evidence for linkage of 3-phosphoglycerate kinase, hypoxanthine-guanine-phosphoribosyltransferase, and glucose 6-phosphate dehydrogenase loci in chinese hamster cells studied by using a relationship between gene multiplicity and enzyme activity. Biochem. Genetics 7, 33-40. 